\title{
Prevalence of caregiver burden, depressive and anxiety symptoms in caregivers of children with psychiatric disorders in Durban, South Africa
}

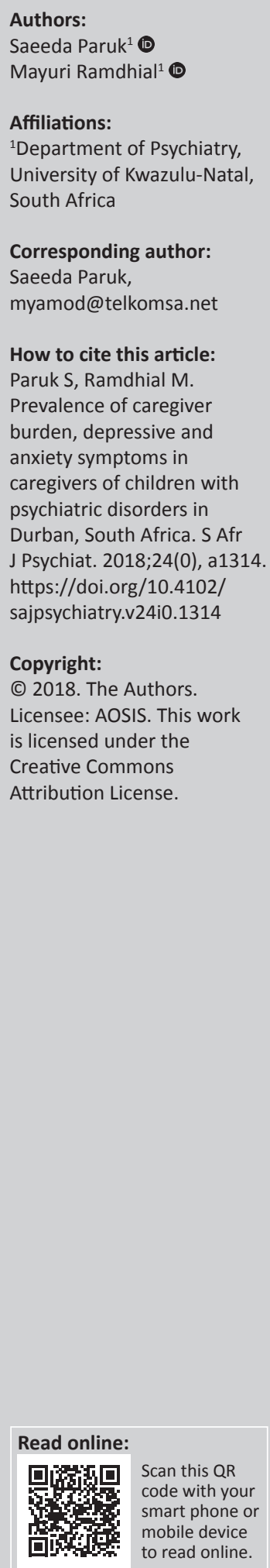

Background: There is increased caregiver burden, depressive and anxiety symptoms associated with the care of mentally ill children. This may be influenced by child or caregiver factors such as socio-demographic and clinical factors and has not been explored in the South African context.

Aim: To describe the prevalence of depression, anxiety symptoms and caregiver burden in caregivers of children treated at psychiatric outpatient services at two public sector hospitals.

Methods: A cross-sectional questionnaire study of 121 adult primary caregivers of children aged 1-17 years with mental illness using a socio-demographic questionnaire, Patient Health Questionnaire (PHQ-9), Generalised Anxiety Disorder-7 Questionnaire (GAD-7), and the Child and Adolescent Impact Assessment (CAIA) to assess caregiver burden.

Results: The caregivers were predominantly female $(n=96,79.5 \%)$ and married $(n=72,59.5 \%)$, with a mean age of $\neg 34.99$ years (SD 10.38), and $74 \%$ were mothers. Among the children, there was a predominance of boys with a 1:4 ratio of girls to boys. The most common diagnoses in the children were attention deficit hyperactivity disorder (ADHD) $(n=56,59.6 \%)$ and autism spectrum disorder $(n=22,23.4 \%)$. Fifty-four $(44 \%)$ caregivers were depressed with a mean PHQ9 score of 5.75 (SD 5.98), and 65 (54\%) reported anxiety symptoms with a mean GAD7 score of 5.71 (SD 5.03). Mothers reported significantly higher levels of anxiety ( $p=0.045$ ) and experienced higher impact on feelings of personal well-being on the CAIA $(p=0.004)$ in comparison with fathers. Caregiver burden was predominantly reported in the domains of restrictions in activities $(n=40,32.8 \%)$, feelings of personal well-being $(n=37,30.7 \%)$ and economic impact $(n=21,17.4 \%)$.

The caregivers of children with ADHD reported higher anxiety levels $(p=0.023)$ than for autistic children. A diagnosis of autistic spectrum disorder was associated with higher income impact $(p=0.004)$ and restrictions impact $(p=0.001)$ than for children with ADHD diagnosis in terms of caregiver burden.

Conclusion: The high prevalence of depression and anxiety symptoms reported amongst caregivers suggests the need for improved mental health screening and psycho-social support programmes for caregivers, particularly mothers. Programmes should consider the impact of caregiving, particularly on mental health, income and social restrictions of caregivers.

Note: A selected abstract from papers presented at the 19th National Congress of the South African Society of Psychiatrists in 'Professional Psychiatric Practice: Medical, Socio-Economic \& Cultural Perspectives', 21-24 September 2018, at the CSIR, Pretoria, South Africa. The congress is hosted by South African Society of Psychiatrists (SASOP). 